\title{
Análisis comparativo del impacto de la transversalidad de la cátedra de emprendimiento en la disposición para nuevos emprendimientos de los alumnos de la Universidad de Guayaquil y la Escuela Superior Politécnica del Litoral
}

\section{Comparative analysis of the impact of the transversality of the chair of entrepreneurship in the provision for new ventures of the students of the University of Guayaquil and the Polytechnic School of the Litoral}

\author{
MAE. María Paulina Brito Ochoa \\ MAE. Luz Elvira Vásquez Luna \\ MAE. Rosa González González \\ MSC. Eva María de Lourdes Loaiza Massuh \\ Universidad de Guayaquil, Ecuador \\ Autor para correspondencia: eva.loaizam@ug.edu.ec, maria.britooch@ug.edu.ec, \\ luz.vasquezlu@ug.edu.ec, rosa.gonzalezg@ug.edu.ec \\ Fecha de recepción: 30 de Agosto de 2016 - Fecha de aceptación: 25 de Octubre de 2016
}

Resumen: Hoy en día el tema de la transferencia de tecnologías y conocimiento por parte de las universidades ecuatorianas para profundizar la investigación científica así como el desarrollo tecnológico y la innovación en el Ecuador es de carácter obligatorio. Por tal motivo las universidades públicas más representativas del Ecuador en la ciudad de Guayaquil, como son la Universidad de Guayaquil y la Escuela Superior Politécnica del Litoral han decidido impartir la cátedra de emprendimiento como un fundamento sólido. Actualmente en la ESPOL la cátedra de Emprendimiento es un curso dirigido para todas las facultades, mientras que en la Universidad de Guayaquil solo se imparte en la Facultad de Ciencias Administrativas. El Objetivo General de la cátedra de emprendimiento tanto en la Universidad de Guayaquil como en la ESPOL es fortalecer la cultura emprendedora en los estudiantes, permitiendo el desarrollo de su creatividad e innovación como elemento dinamizador en la generación de ideas y planes de negocios que conlleven a futuro a la creación de empresas sólidas y competitivas que brinden plazas de trabajo, permitiendo cambios importantes en el desarrollo y bienestar de la sociedad. El presente estudio es de tipo cuasi experimental con una asignación aleatoria de estudiantes que se encuentran tomando la materia y estudiantes que como parte de su malla han estudiado alguna vez la materia. En ESPOL se han considerado a estudiantes que hayan cumplido con más del $50 \%$ de su malla y en el caso de la Universidad de Guayaquil, se encuestó a estudiantes del 5to semestre (viendo la materia) y estudiantes del 8vo semestre (terminando sus carreras). Esta investigación realiza un análisis comparativo entre la Universidad de Guayaquil y la ESPOL sobre el impacto que tiene el impartir la cátedra de emprendimiento en la disposición de generar emprendimientos por parte de los estudiantes, la aplicabilidad de los conocimientos adquiridos por los estudiantes al momento de emprender y el identificar cuáles son factores principales que impiden mayormente la generación de ideas o planes de negocios. Con los resultados obtenidos en el presente estudio, se espera proponer argumentos sólidos para generar una propuesta de transversalidad de la cátedra de emprendimiento aplicable a las 18 Facultades con que cuenta la Universidad de Guayaquil, ya que son las universidades, los agentes formadores y transformadores que deben promover en sus estudiantes la cultura emprendedora. 
Palabras claves: emprendimiento; transferencia de conocimiento; creatividad; innovación

Abstract: Currently the issue of technologies and knowledge transfer by universities in Ecuador to deepen scientific research as well as technological development and innovation is mandatory. For this reason the most representative public universities of Ecuador in Guayaquil city, such as the University of Guayaquil and the Escuela Superior Politécnica del Litoral have decided to teach the subject of entrepreneurship as a solid foundation. Nowadays in ESPOL the subject of entrepreneurship is a course directed to all faculties, while at the University of Guayaquil is only taught in the Faculty of Administrative Sciences. The General objective of the entrepreneurship subject in both at the University of Guayaquil as at ESPOL is strengthening the entrepreneurial culture in students, allowing the development of their creativity and innovation as a dynamic element in the generation of ideas and business plans that lead them to a future where strong and competitive companies that provide jobs are created by them, allowing important changes in the development and welfare of the society. The present study corresponds to the quasi experimental type with a random allocation of students that are taking the subject and students that as part of their curriculum have studied any time the subject. At ESPOL there were considered students who have completed more than $50 \%$ of the curriculum and in the case of the University of Guayaquil, the survey was carried on among students from the 5th semester (taking the subject at the present) and students of the 8th semester (ending their careers). This research makes a comparative analysis between the University of Guayaquil and the ESPOL about the impact that teaching the entrepreneurship subject has over the students' purpose of generating projects, the applicability of the acquired knowledge by students at the moment to undertake and identify main factors that mostly prevent the generation of ideas or business plans. With the obtained results in the present study, is expected to propose strong arguments to generate a proposal of transversally teaching the entrepreneurship subject applicable to the 18 Faculties of the University of Guayaquil, since universities are the training agents and transformers that must promote to their students the entrepreneurial culture.

Key words: entrepreneurship; transfer of knowledge; creativity and innovation

\section{Introducción}

Las condiciones económicas actuales, evidentes para todos y corroboradas por los datos de Kelly, Singer, \& Herrington (2015) en el informe GEM donde determinaron que el 15,8\% de la población en el Ecuador que actualmente se encuentra desempleada se ha decidido a emprender. Esta realidad deja ver la palpable necesidad de generar fuentes de empleo como condición sine qua non para combatir la crisis, es aquí donde la Universidad, se convierte en puente importante entre ambas.

Es la Universidad al momento de transferir conocimiento a los futuros profesionales que en sus aulas se forman la llamada a infundir a sus estudiantes el mirar la crisis como una oportunidad de crecimiento, ya que es bien conocido que durante tiempos de crisis económica es cuando mayormente la creatividad aflora, aquí es cuando nacen los nuevos negocios o nuevos emprendimientos.

La Universidad es generadora e impartidora de herramientas que permitirán la correcta iniciación de emprendimientos, así como las directrices para que estos emprendimientos se sostengan y perduren en el tiempo. En la Universidad de Guayaquil se imparte la cátedra de emprendimiento únicamente en la Facultad de Ciencias Administrativas mientras que en la ESPOL se lo hace en mayor número de Facultades, esta realidad nos conduje a realizar el presente análisis comparativo entre la realidad de los estudiantes que reciben la cátedra en ambas Instituciones de 
Educación Superior con la finalidad de medir el impacto de las herramientas que desde la academia se ha entregado al momento de emprender y al momento de tomar decisiones para resolver situaciones durante el desarrollo del emprendimiento.

\section{Revisión de literatura}

\section{Características del Emprendedor}

Históricamente se define al emprendedor como un agente visionario que aceptaba el riesgo de comprar a precios ciertos y vender a precios inciertos como lo indica Cantillón, en 1755 siendo, citado entre otros por Cardozo (2010) y Van den Berg (2012); o el manejado por Schumpeter en 1943 que lo define como quien implementa nuevas combinaciones de medios de producción, en un rol innovador y como figura en el desarrollo económico ( (Yoguel, Barletta, \& Pereira, 2013); (Cardozo, 2010)).

El Global Entrepreneurship Monitor (GEM), lo define como "Cualquier intento de crear un nuevo negocio o empresa, tales como autoempleo, una nueva organización empresarial, o la expansión de un negocio existente, por un individuo, un equipo de personas, o un negocio establecido" (Reynolds et al., 1999, p. 3, en Singer, 2014).

\section{Emprendedores Universitarios}

Varios negocios con impacto a nivel global han sido iniciados por estudiantes universitarios como FedEx, Dell, Google, Facebook y Snapchat (Zhang, Wang y Owen, 2015). Adicionalmente, existe evidencia del impacto de las universidades en el emprendimiento gracias a indicadores como la proliferación de oficinas de transferencia de tecnología, incremento de patentes y licencias, así como cambios en la misión, criterios de permanencia y promoción de las universidades (Goldstein, 2010)

Dado que el estudio se realiza sobre estudiantes universitarios, que tienen cursos de emprendedores, es importante considerar a Küttim, Kallaste, Venesaar, \& Kiis (2014) que muestra que los estudiantes valoran mucho las redes de contacto y el apoyo en el emprendimiento

Según el estudio realizado por los autores, Ismail, Zain, \& Zulihar (2015), se puede mostrar que la educación emprendedora juega un rol importante en la aparición y crecimiento de nuevos emprendedores. Por esta razón es sumamente importante desarrollar las competencias emprendedoras con el fin de que sean emprendedores exitosos para el bien del país (Martínez De Luco \& Campos Granados, 2014)

Ismail, Zain, \& Zulihar (2015) recomiendan que para las instituciones que implementan la educación en emprendedurismo, debe evaluarse la efectividad de las competencias emprendedoras durante el programa. A nivel internacional, una de las variables de análisis es el género; las investigaciones de Ventura \& Quero (2013) indican que la intención de emprender en las mujeres está relacionada en forma directa con su autoconfianza. Para Maes, Leroy, \& Sels (2014), sugieren que la mujer para emprender debe compatibilizar su emprendimiento con su vida familiar y lo que 
más despierta la intención de emprender es la necesidad de identificarse con mujeres emprendedoras.

\section{Intención emprendedora Universitaria}

De acuerdo con Krueger (2008), las intenciones son consideradas como el mejor predictor del comportamiento humano. Yang (2013) verifica en su estudio sobre estudiantes universitarios que la teoría del comportamiento planificado (TPB- Theory of Planned Behavior) según Ajzen, 1991, se puede vincular con la intención emprendedora de los estudiantes y sus actitudes. Del mismo modo, Serra \& Kabadayi (2014) relacionan la educación con la intención emprendedora como predictora de futuros emprendedores.

\section{Objetivo.-}

Con este estudio se pretende analizar el nivel de intensión emprendedora de los alumnos de la Universidad de Guayaquil y de la Escuela Superior Politécnica que se encuentran recibiendo la cátedra de Emprendimiento.

\section{Objetivos específicos:}

- Identificar el grado de emprendimiento previo realizado por los estudiantes de la cátedra emprendedores.

- Identificar el nivel de disposición para el emprendimiento en los estudiantes que se encuentran a un nivel de 50\% de su avance en la carrera y además están recibiendo la cátedra emprendedora.

- Realizar una comparación con los resultados de las dos universidades objeto de estudio

- Analizar la percepción de la aplicabilidad de la cátedra Emprendedores en el desenvolvimiento de los emprendimientos de los alumnos

- Identificar los factores principales que los alumnos perciben como impedimento para iniciar su propio emprendimiento

\section{Metodología}

Este trabajo tiene un enfoque cuantitativo, transversal, de tipo descriptivo; la herramienta para el levantamiento de la información fue la encuesta, aplicado tanto por la Universidad de Guayaquil como por la Espol, en el periodo de Enero a Junio 2016.

La encuesta fue autoaplicada, mediante preguntas abiertas, cerradas, de opción múltiple y con escala de liker. Inicia con preguntas generales y luego se encamina hacia las particulares y específicas, donde se incluye la intención de emprendimiento y el tipo de negocio en el cual el estudiante desearía incursionar.

Se anexa una tabla en la que resumen la cantidad de estudiantes encuestados, sus características principales y el número de encuestas aplicadas tanto en la Universidad de Guayaquil como en la Escuela Superior Politécnica del Litoral, ambas instituciones públicas, y representativas entre las más grandes ubicadas en la ciudad de Guayaquil. 
Tabla1. Descripción del levantamiento de información mediante encuestas.

\begin{tabular}{cll}
\hline Universidad & Universidad de Guayaquil (UG) & $\begin{array}{c}\text { Escuela } \\
\text { Litoral } \\
\text { (ESPOL) }\end{array}$ \\
\hline Característica & $\begin{array}{l}\text { Estudiantes de 5to semestre, cursando la cátedra } \\
\text { de emprendimiento, de la Facultad de Ciencias }\end{array}$ & $\begin{array}{l}\text { Estudiantes de 5to semestre, cursando } \\
\text { la cátedra emprendedora. A nivel de } \\
\text { todas las carreras de la institución } \\
\text { Administrativas, que involucran 8 carreras }\end{array}$ \\
& 869 & 139 \\
\hline
\end{tabular}

Fuente: Elaboración propia

La encuesta se realizó de forma anónima para incentivar la veracidad de la información otorgada por los estudiantes. Para el caso de la UG fue realizada mediante encuesta impresa, y en la ESPOL mediante la herramienta de google drive.

\section{Resultados}

Tal como fue estructurada la encuesta se presentan a continuación los principales resultados que han sido considerados en las dos universidades objeto de estudio para el presente trabajo. Se consideró además los análisis por cada apartado investigado. Cabe recalcar que el instrumento utilizado fue exactamente el mismo en ambas universidades, con la particularidad de que en la UG se realizó mediante encuesta física facilitada y en la Espol se utilizó la herramienta Google Drive.

El periodo de levantamiento de información se dio de Enero a Junio del 2016, a los alumnos que se encontraron cursando la cátedra de emprendimiento en ambas universidades.

\section{Datos generales}

\section{1.- Rangos de Edad}

Encontramos en los rangos de edad de los alumnos de la UG, si bien existe un rango amplio de la edad que va desde los 18 años hasta más allá de 50 años, la concentración se encuentra entre las edades de 20 y 23 (73\%), la Espol mantiene un comportamiento bastante similar en los rangos de edad, con un $73 \%$ en edades entre 20 y 23 años.

Una tendencia encontrada en las carreras de la UG en el área administrativa, es que la presencia del género femenino es mayoritaria, mientras la presencia del género masculino es más frecuente en las carreras de la ESPOL. Como podemos observar en los siguientes gráficos tanto la UG como la ESPOL consideran la incursión del género como elemento aleatorio del estudio. 


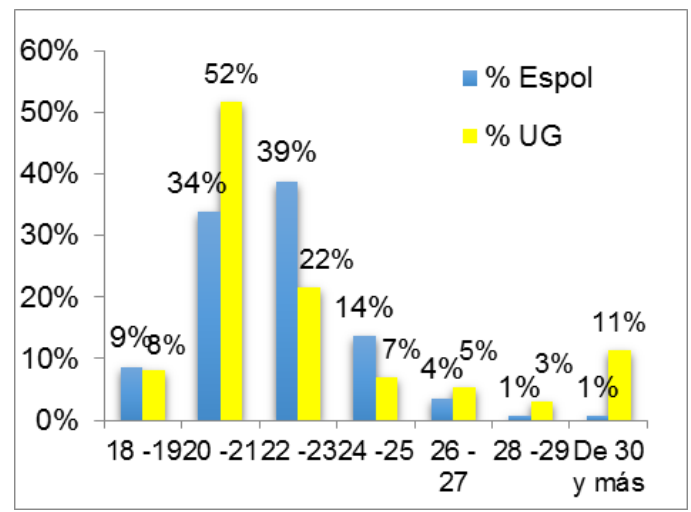

Figura 1. Edad alumnos UG

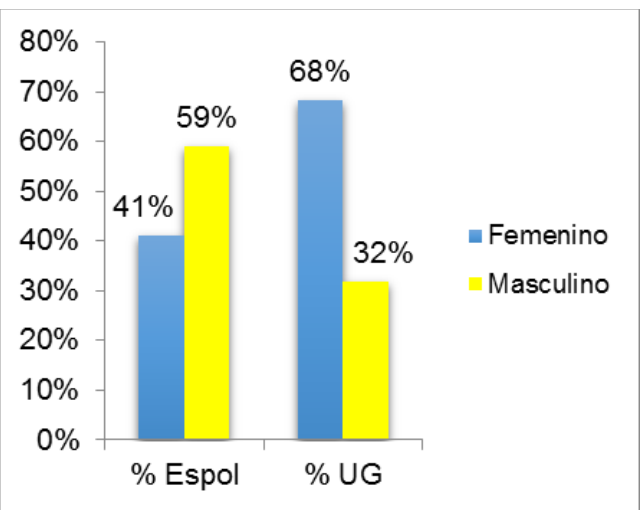

Figura 2. Género alumnos ESPOL

\section{2.- Iniciativa emprendedora del estudiante previo a la cátedra de emprendimiento}

Es conocido que no se requiere de conocimientos académicos para iniciar un emprendimiento, y esta hipótesis ha sido corroborada ya que los alumnos de ambas universidades, que sin haber obtenido un título universitario han incursionado de forma empírica en algún tipo de negocio. En la UG es aproximadamente 3 de cada 10 alumnos los que han experimentado esta faceta y en la ESPOL 2 de cada 10.

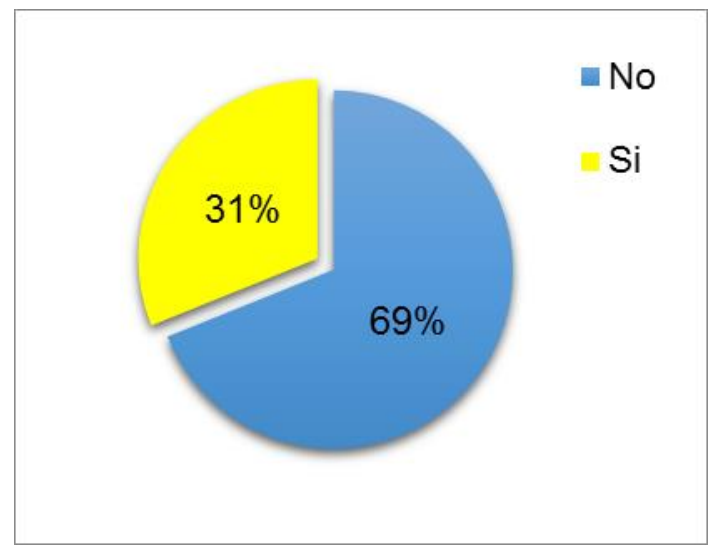

Figura 3. Han emprendido antes - UG

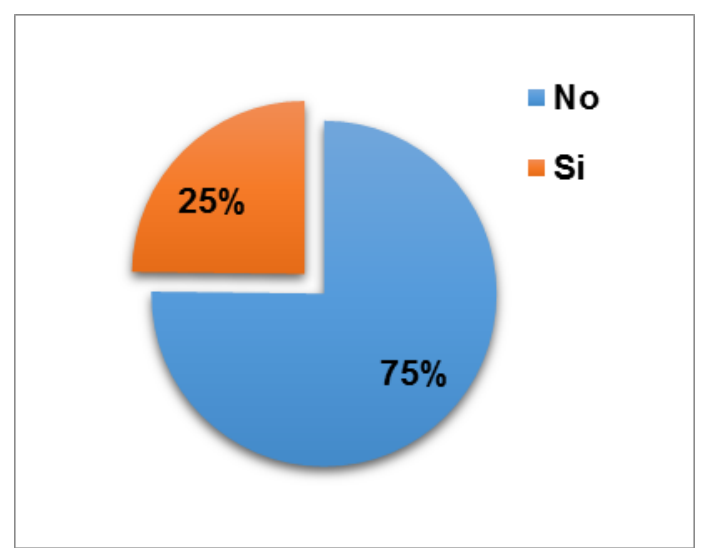

Figura 4. Han emprendido antes - ESPOL

\section{3.- Nivel de percepción de la aplicabilidad de la cátedra emprendedora en los negocios de los alumnos}

De los alumnos que han incursionado en emprendimiento, se encontró que el 85\% de alumnos en la UG señalan que la cátedra de emprendimiento ha sido de ayuda para mejorar sus negocios, sin embargo podemos observar que los alumnos de la ESPOL un 55\% indican haber aplicado los conocimientos adquiridos en su carrera como ayuda en sus emprendimientos o negocios. 


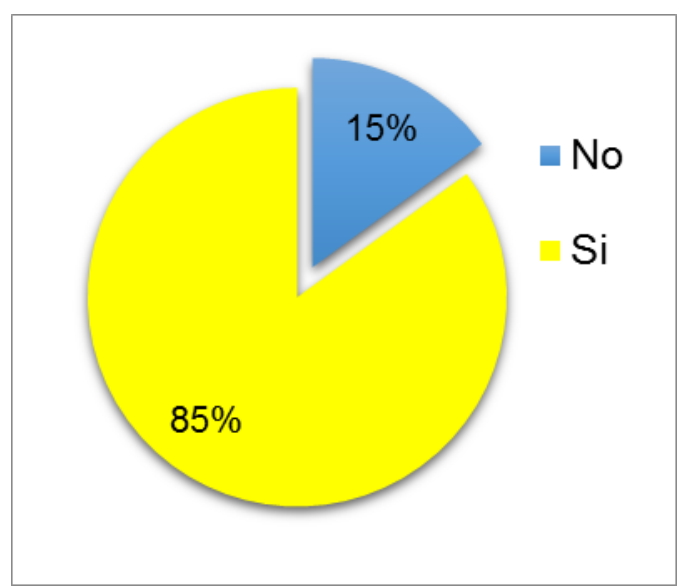

Gráfico 5. Aplicabilidad de la cátedra UG Fuente: Elaboración propia

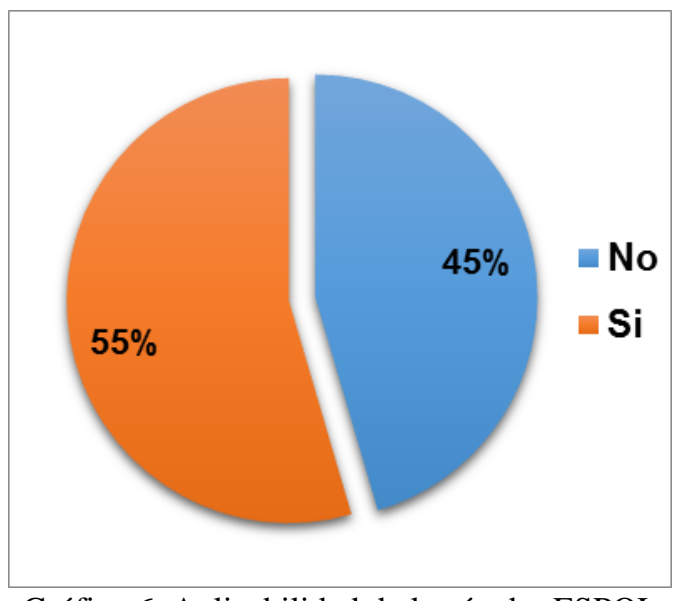

Gráfico 6. Aplicabilidad de la cátedra ESPOL Fuente: Elaboración propia

\section{4.- Nivel de intención emprendedora de los alumnos de emprendimiento}

La mayoría de los alumnos que se han estado recibiendo la cátedra Emprendedores menciona encontrarse dispuesto a iniciar su propio negocio o empresa, es el caso tanto en la UG como en la ESPOL con porcentajes de $98 \%$ y $93 \%$ respectivamente.

Existe motivación en los alumnos para entrar en el campo del emprendimiento, comprendiendo la dificultad que amerita en muchos casos emprender un negocio en la situación económica actual, sin embargo reconocen las circunstancias que se pueden generar producto de la correcta identificación de oportunidades.

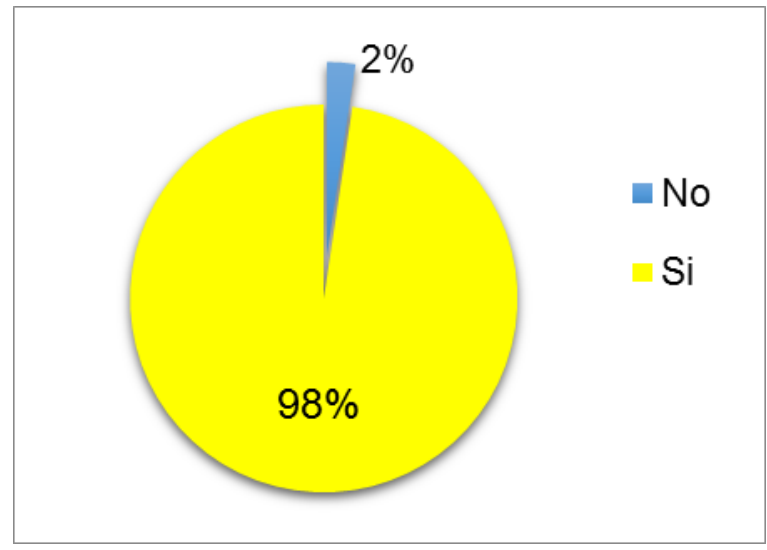

Figura 7. Intensión de Emprender UG Fuente: Elaboración propia

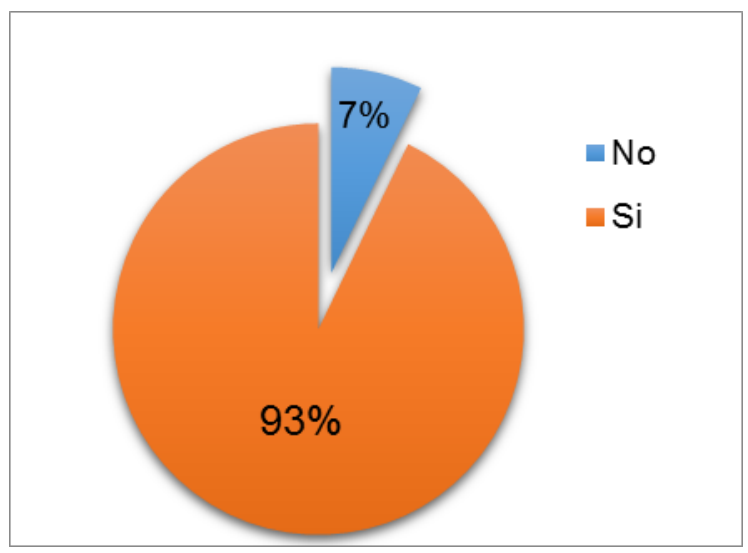

Figura 8. Intención de Emprender ESPOL Fuente: Elaboración propia

\section{5.- Nivel de percepción de la capacidad que tienen los alumnos para el emprendimiento}

Cabe recalcar que esta pregunta fue realizada en una escala de Likert que va del 1 al 10, donde 10 significa encontrarse altamente capacitado y 1 nada capacitado. Se realizó un promedio ponderado con las respuestas de ambas universidades y los alumnos de la Espol manifiestan encontrarse capacitados en un promedio de 6,83/10, mientras los alumnos de la UG promedian un $6,49 / 10$. 
A pesar de la alta disposición al emprendimiento que los alumnos manifiestan en la pregunta anterior, se puede evidenciar que la gran mayoría no se sienten muy capacitados $(8,9$ y 10), se identificó que en la Espol un 36,5\% se considera bastante capacitado y en la UG un 29,5\% apenas lo considera de esa forma. Son varios los factores que afectan este criterio, y se reflejaron como falta de experiencia, dificultades para encontrar financiamiento, entre otras.

\section{6.- Disposición a Emprender un negocio ahora}

La relación de disposición a emprender un negocio en la actualidad para los alumnos de la UG se encuentra un poco más marcada en referencia a los alumnos de la Espol.

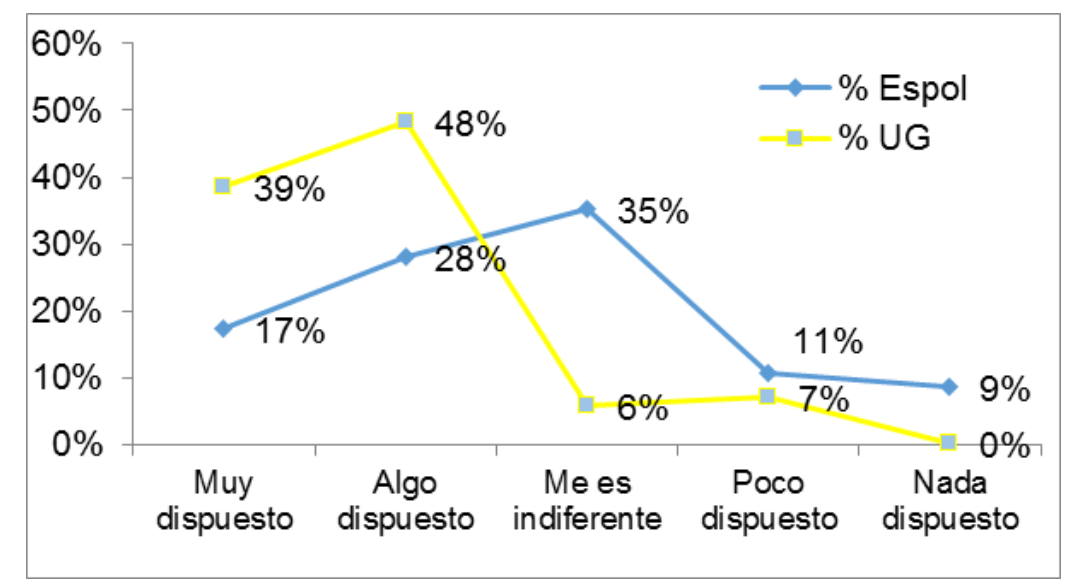

Figura 9. Emprendería ahora UG vs ESPOL

Fuente: Elaboración propia

\section{7.- Disposición a iniciar un emprendimiento en el mediano y largo plazo.}

Se halló que es mayor el porcentaje de la disposición de iniciar un emprendimiento en el mediano y largo plazo, esto es de 3 a 5 años después, podría considerarse que los alumnos, al no sentirse muy capacitados para emprender, prefieren adquirir un poco más de conocimiento y experiencia para enfrentar las dificultades que conlleva iniciar un negocio.

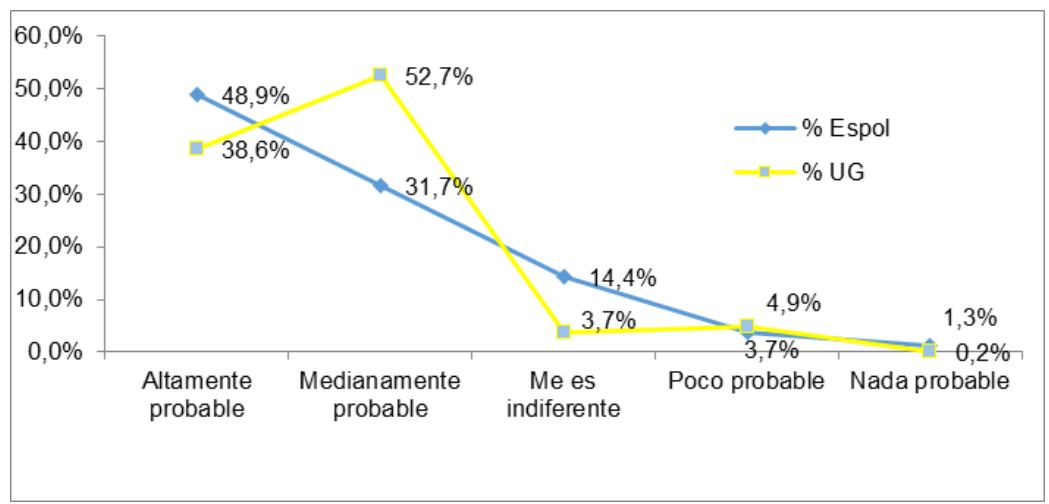

Figura 10. Emprendería dentro de 3 a 5 años - UG vs ESPOL Fuente: Elaboración propia 


\section{8.- Causas por las que no emprenderían un negocio ahora}

Las principales causas por las cuales los alumnos mencionan que no emprenderían en este momento se dan por factores económicos, como la economía del país y la escasez del dinero. La relación de motivaciones al no emprendimiento se dan de forma bastante parecida entre ambas universidades.

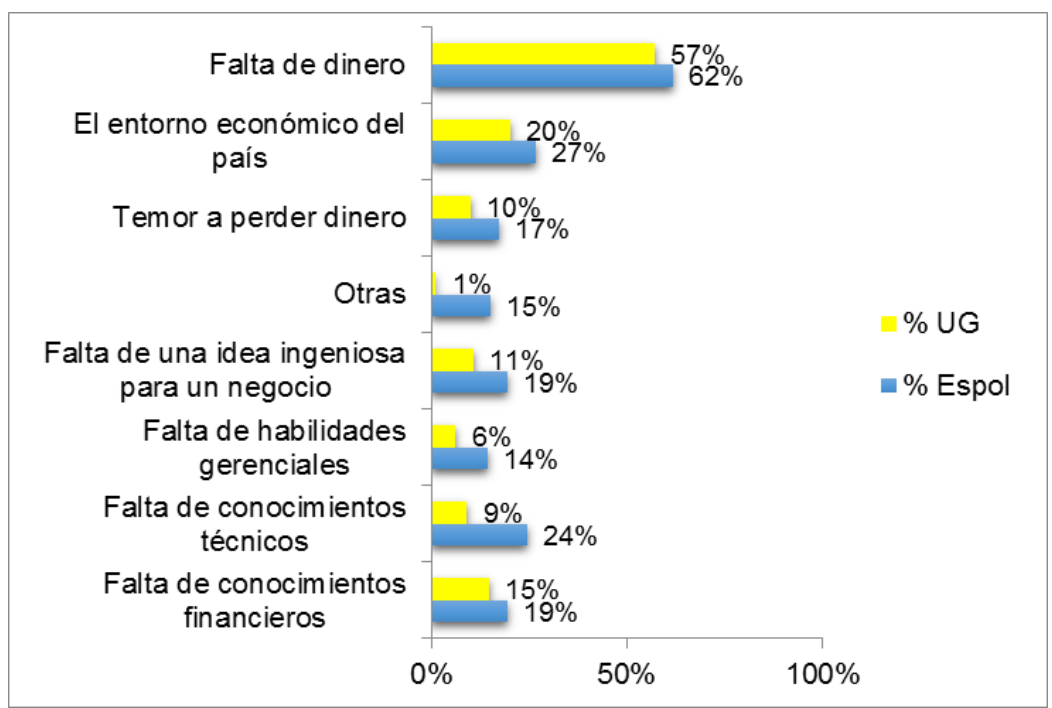

Figura 11. Causas para no Emprender en la actualidad - UG vs ESPOL Fuente: Elaboración propia

\section{Discusión}

La intención de emprendimiento se da de forma natural para muchas personas, y sabemos además que no siempre resultan en negocios efectivos ya sea por los métodos como por las habilidades del emprendedor. En este estudio se analiza el nivel de emprendimiento de los alumnos que por su propia iniciativa empezaron un negocio y de qué forma el recibir la cátedra de emprendimiento influye sobre la motivación para iniciar su propio negocio (Maluk Salem, 2014).

En base en lo expuesto, encontramos que alrededor de 3 de cada 10 alumnos en promedio para ambas universidades de la ciudad de Guayaquil, tuvo la iniciativa de emprender su propio negocio, y de ellos la mayoría provenían de actividades en conjunto con la familia, también hay que tener en cuenta que no son porcentajes significativos porque no se incluyeron en el estudio otras variables como las metas personales y familiares, el nivel de ingreso de las familias o las edades específicas que aún están bajo la tutela de sus padres.

No obstante de estos emprendedores un $85 \%$ de los alumnos de la UG manifestaron que la cátedra les ha resultado de ayuda para guiar o manejar sus negocios, los alumnos de la Espol también manifiestan en un $55 \%$ que la cátedra universitaria le ha servido de guía en sus emprendimientos. 
Es importante destacar este punto de la formación académica, dado que las universidades están encaminadas a colaborar en la construcción de negocios eficientes, en formar para fortalecer las empresas del medio, a través de la innovación de productos y servicios, mediante herramientas que le sean útiles para analizar las diferentes estrategias de mercado y de producto. Si bien muchos negocios inician con la necesidad de generar un empleo propio, que no nace de lo académico, se reconoce la importancia de este rol formal para fortalecer las ideas de negocios que se construyen basados en un modelo de valor y eficiencia (Vasquez , Loaiza Masshu, \& González González, 2016).

Una vez que los alumnos han cursado la mitad de su carrera universitaria, se encuentran con la materia Emprendimiento dentro de su malla, y es aquí donde se hace un énfasis en la preparación académica para hacer frente a la creación de un negocio rentable y eficiente. La cátedra pretende además de motivar a los alumnos para que tengan la confianza de incursionar en emprendimiento, facilitarle herramientas útiles que les permitan enfrentarse a la competitividad del mercado.

Es mayoritario el porcentaje de alumnos que se encuentran motivados a iniciar un negocio propio, sin embargo a pesar de este nivel de disposición, los mismos alumnos consideran aún no se encuentran suficientemente capacitados para iniciar su negocio. La comprensión de la complejidad de manejar un negocio, se reconoce como riesgosa por el nivel de variables no controlables que existen en el mercado. Es quizás por esto que su disposición de emprender no se ve reflejada en el corto plazo, sino más bien en el mediano y largo plazo, es decir de 3 a 5 años, esto se puso evidenciar de forma muy similar entre las dos universidades.

Finalmente, el grado de incertidumbre de los alumnos acerca de los factores económicos, así como de la falta de dinero propio, son mencionados como las principales causas que frenarían su iniciativa de emprendimiento. Considerando la disposición a emprender en el mediano plazo, se podría hacer otro estudio con estudiantes que han finalizado sus carreras.

\section{Conclusiones}

Se puede concluir lo siguiente:

De las variables estudiadas se puede evidenciar que es relativamente alta la percepción de los alumnos respecto a la ayuda que les brinda la cátedra emprendimiento en sus negocios propios.

Es este estudio se demuestra la importancia de que en las universidades se brinde formación en temas de emprendimiento para el manejo adecuado de los negocios ya que ejercen una gran influencia en las personas que desean ser los dueños de sus propios negocios.

La necesidad latente que existe en los alumnos de seguir capacitándose en temas administrativos, financieros, legales que les permitan tener mayor seguridad para emprender y afrontar exitosamente dificultades que los lleven a mantener negocios estables que perduren en el tiempo.

\section{Bibliografía}


Cardozo, A. (2010). La Motivación para Emprender. Evolución del Modelo de Rol en Emprendedores Argentinos. Universidad Nacional de Educación a Distancia.

CLADEA. (2015). Universidad de Valparaíso Chile. Recuperado el 2016, de http://www.faceauv.cl/cladea2015/images/stories/track5/CLADEA_2015_submission_39 6.pdf

GEM. (2015). Espae. Recuperado el 2016, de http://www.espae.espol.edu.ec/images/documentos/publicaciones/libros/gemecuador2014 .pdf

Goldstein, H. (2010). The 'entrepreneurial turn' and regional economic development mission of universities. Annals of Regional Science, 83-109.

Ismail, V. Y., Zain, E., \& Zulihar. (2015). The Portrait of Entrepreneurial Competence on Student Entrepreneurs. Procedia. Social and Behavioral Science, 169, 178-188.

Krueger, N. (08 de 06 de 2008). http://papers.ssrn.com/. Recuperado el 10 de 03 de 2016, de Entrepreneurial Resilience: real \& perceived barriers to implementing entrepreneurial intentions.: http://papers.ssrn.com/sol3/papers.cfm?abstract_id=1155269

Küttim, M., Kallaste, M., Venesaar, U., \& Kiis, A. (2014). Entrepreneurship education at university level ans students' entrepreneurial intentions. Procedia. Social and Behavior Sciences, 110, 658-668.

Maes, J., Leroy, H., \& Sels, L. (2014). Gender differences in entrepreneurial intentions: A TPB multi-group analysis at factor and indicator level. European Management Journal, 32, 784794.

Maluk Salem, O. (2014). Congreso Latinoamericano de Ciencia, Tecnología, Innovación y Educación. Recuperado el 2016, de OIE: www.oei.es/congreso2014/memoriactei/180.pdf

Martínez De Luco, G., \& Campos Granados, J. (2014). La intención emprendedora en estudiantes universitarios: El caso de la Universidad de Deusto. BizcaiLab.

Mustapha, M., \& Selvaraju, M. (2015). Personal Attributes, Family Influences, Entrepreneurship Education and Entrepreneurship Inclination Among University Students. Kajian Malaysia, 33(1), 155-172.

Serra, E., \& Kabadayi, Z. D. (2014). Exploring the antecedents of entrepreneurial intention on Turkish university studens. Procedia. Social and Behavior Sciences, 150, 841-850.

Van den Berg, R. (2012). 'Something Wonderful and Incomprehensible in Their Oeconomy': The English Versions of Richard Cantillon's Essay on the Nature of Trade in General. European Journal Of The History Of Economic Thought, 19(6), 868-907. 
Vasquez , L. E., Loaiza Masshu, E., \& González González, R. (2016). Tranversalidad de la cátedra Emprendedores en la UG. Guayaquil.

Ventura, R., \& Quero, M. (2013). Factores explicativos de la intención de emprender en la mujer. Aspectos diferenciales en la población universitaria según la variable de género. Cuadernos de Gestión, 13(1), 127-149.

Yang, J. (2013). The theory of planned behavior and prediction of entrepreneurial intention among chinese undergraduates. Social Behavior \& Personality, 41(3), 367-376.

Yoguel, G., Barletta, F., \& Pereira, M. (jul-set de 2013). De Schumpeter a los Poschumpeterianos: Viejas y Nuevas Dimensiones Analíticas. Problemas del Desarrollo. Revista Latinoamericana de Economía., 174, 35-59. 